\title{
Os impactos da pandemia de COVID-19 na saúde mental da população
}

The impacts of the COVID-19 pandemic on the mental health of the population

Los impactos de la pandemia COVID-19 en la salud mental de la población

Isadora Correia Dias ${ }^{1 *}$, Camila Hibner de Almeida ${ }^{1}$, Érika Mendonça Martins Melo ${ }^{1}$, Hemilly Costa Dias $^{2}$, Isabella Soares Luz ${ }^{1}$, João Luiz Duarte Santos ${ }^{3}$, Júlia Ferraz Barbosa1, Luana Favoreto Zanetti ${ }^{1}$, Rodrigo Machado Novais Filho ${ }^{1}$, Gustavo Fonseca Genelhu Soares ${ }^{4}$.

\section{RESUMO}

Objetivo: Discutir o aumento na incidência de depressão e ansiedade na população durante o período de pandemia da COVID-19, a partir de análise da produção científica sobre o tema. Revisão bibliográfica: A pandemia da COVID-19 tem impactado na saúde e no cotidiano das pessoas. Medidas para conter a transmissão do vírus alteraram o modo de se portar, promoveram o constante uso de máscaras, a necessidade de distanciamento social e a diminuição de contatos e relações interpessoais, repercutindo na saúde mental e desenvolvimento de doenças como a ansiedade e a depressão na população. Como consequência, tem se observado aumento do consumo de antidepressivos e de outras substâncias como: álcool, cigarro e outras drogas. Salienta-se, ainda, o impacto psicossocial nos profissionais de saúde, sobretudo naqueles que atuam na linha de frente no enfrentamento da pandemia. Considerações finais: É de suma importância que haja um engajamento entre os órgãos reguladores, as intuições de saúde e também da população para gerenciar não só o impacto direto da pandemia na saúde, mas também todos os fatores secundários que têm contribuído para o adoecimento mental das pessoas nesse período.

Palavras-chave: Infecções por coronavirus, Depressão, Saúde mental.

\begin{abstract}
Objective: To discuss the increase in the incidence of depression and anxiety in the population during the COVID-19 pandemic period, based on an analysis of the scientific production on the subject. Bibliographic review: The COVID-19 pandemic has impacted people's health and daily lives. Policies to contain the transmission of the virus has changed our behaviors by promoting the constant use of masks, the need for social distance, and the reduction of contacts and interpersonal relationships. All of this affected mental health and increased the development of diseases such as anxiety and depression in the population. As a consequence, there has been an increase in the consumption of antidepressants and other substances such as alcohol, cigarettes, and other drugs. It also has emphasized the psychosocial impact on health professionals, especially those who work on the front line, fighting against the pandemic. Final considerations: It is of utmost importance that there is an engagement between the government, the health intuitions, and also the population to manage not only the direct impact of the pandemic on the health but also manage all the secondary factors that have contributed to increasing the mental health diseases on people.
\end{abstract}

Key words: Coronavirus infections, Depression, Mental health.

\footnotetext{
${ }^{1}$ Centro Universitário UNIFAMINAS (UNIFAMINAS), Muriaé - MG.

*E-mail: isadoracorreeia@yahoo.com.br

${ }^{2}$ Faculdade de Medicina do Vale do Aço (UNIVAÇO), Ipatinga - MG.

${ }^{3}$ Faculdade de Minas (FAMINAS), Belo Horizonte - MG

${ }^{4}$ Centro Universitário de Caratinga (UNEC), Caratinga - MG.
}

SUBMETIDO EM: 6/2021

ACEITO EM: 6/2021

PUBLICADO EM: 7/2021 


\section{RESUMEN}

Objetivo: Discutir el aumento de la incidencia de depresión y ansiedad en la población durante el período pandémico del COVID-19, a partir de un análisis de la producción científica sobre el tema. Revisión bibliográfica: La pandemia de COVID-19 ha tenido un impacto en la salud y en la vida de las personas. Las medidas para contener la transmisión del virus cambiaron la forma de comportarse, promovieron el uso constante de máscaras, la necesidad de distanciamiento social y la reducción de contactos y relaciones interpersonales, afectando la salud mental y el desarrollo de enfermedades como la ansiedad y la depresión en la población. Como consecuencia, es posible observar un aumento en el consumo de antidepresivos y otras sustancias como alcohol, cigarrillos y otras drogas. También enfatiza el impacto psicosocial en los profesionales de la salud, especialmente aquellos que trabajan en primera línea para enfrentar la pandemia.

Consideraciones finales: Es de suma importancia que exista un compromiso entre los órganos reguladores, las intuiciones sanitarias y también la población para gestionar no solo el impacto directo de la pandemia en la salud, sino también todos los factores secundarios que contribuyen a la aparición y agravamiento de enfermedades mentales en este período.

Palabras clave: Infecciones por coronavirus, Depresión, Salud mental.

\section{INTRODUÇÃO}

Em dezembro de 2019, na China, surgiu uma nova cepa de coronavírus denominada coronavírus da Síndrome Respiratória Aguda Grave 2 (SARS-CoV-2), que se espalhou mundialmente e, em março de 2020, foi considerada uma pandemia pela Organização Mundial da Saúde (OMS). O coronavírus 2019 (COVID-19) se tornou, então, uma emergência internacional de saúde pública, levando os países a tomarem medidas de prevenção contra um vírus que consegue se alastrar rapidamente (BRASIL, 2020; SILVA HN, et al., 2020).

Inicialmente, acreditava-se que o vírus causava mais complicações em pessoas com o sistema imune comprometido, como os idosos e pessoas que apresentavam doenças crônicas e imunossupressoras. A infecção por esse vírus pode causar desde sintomas clínicos respiratórios semelhantes a uma gripe simples até a uma pneumonia atípica, podendo levar à morte. Outros sintomas comumente associados são a perda de paladar e olfato e sintomas menos comuns como náuseas, diarreia, alteração do nível de consciência e lesões de pele. Entretanto, por ser uma doença nova e de rápida mutação, os estudos para compreendê-la melhor continuam (FIOCRUZ, 2020).

Com o intuito de evitar a disseminação descontrolada da doença, foram adotadas medidas de prevenção como o isolamento dos casos suspeitos, o distanciamento social, o uso de máscara, lavagem frequente das mãos, limpeza dos ambientes e evitar o compartilhamento de objetos (BRASIL, 2020). Porém, com as pessoas ficando mais tempo nos domicílios, o aumento da jornada de trabalho, preocupações com os filhos que também estão estudando em casa, atritos matrimoniais, aumento no número de casos e de óbitos, entre outras mudanças, contribuiu para o aumento de problemas nas esferas sociais, econômicas, educacionais, físicas e psicológicas dos indivíduos (SILVA HN, et al., 2020).

Muitas pessoas desenvolveram doenças como ansiedade e depressão durante a pandemia. A depressão é uma doença psiquiátrica de cunho muito sério, crônico e pode ser confundida com a ansiedade, caracterizada pela tristeza persistente e perda da vontade de cumprir as atividades que proporcionam prazer, oscilações de humor e até pensamentos suicidas. Com o aumento dessas doenças psíquicas, aumentou também o consumo de antidepressivos de primeira linha que atuam no controle de neurotransmissores do Sistema Nervoso Central (SNC) e também o uso de álcool, cigarro e outras drogas para lidarem melhor com as emoções. Segundo a OMS, o Brasil está atrás somente dos Estados Unidos no ranking de países com mais pacientes com diagnóstico de depressão, e em primeiro lugar em quadros de ansiedade (FIOCRUZ, 2020).

Assim, observou-se que as medidas de prevenção adotadas para o controle do número de casos de COVID-19 geraram grandes impactos na saúde mental da população de forma geral. Os principais sintomas 
psicopatológicos causados pela quarentena são medo, ansiedade, humor deprimido, maiores chances de abuso alcoólico, estresse pós-traumático e depressão. Esse quadro resulta de um conjunto de fatores que vão além da redução do contato com outras pessoas, mas também por questões econômicas, pelo medo da doença e até mesmo pelo impedimento de se despedir daqueles que morrem (BROOKS SK, et al., 2020).

Há, também, um grande volume de informações falsas em redes sociais, sem base científica, notícias negativas, afetando gradativamente a saúde mental dos indivíduos. Os humanos são seres sociáveis e que possuem necessidade de contato físico e social como abraçar, consolar e cuidar daqueles que sofrem. Ao retirar isso que é inato e natural ao homem, inevitavelmente, surgirão consequências psicológicas (AFONSO $\mathrm{P}, 2020)$.

Assim, é de suma importância a adoção de medidas que visem a redução dos impactos psicológicos, principalmente o índice de depressão, que a pandemia e suas medidas preventivas têm desencadeado na população (SCHMIDT B, et al., 2020). Diante disso, o presente estudo tem por objetivo discutir o aumento na incidência de depressão e ansiedade na população durante o período de pandemia da COVID-19, a partir de análise da produção científica atual sobre o tema.

\section{REVISÃO BIBLIOGRÁFICA}

Atualmente, a pandemia do novo coronavírus (SARS-CoV-2) continua gerando preocupação em autoridades sanitárias e na população do mundo todo (OMS, 2020). Visto que a principal forma de transmissão da COVID-19 ocorre através de gotículas respiratórias emitidas por pacientes infectados e por contato direto com os mesmos, foram necessárias diversas medidas de restrição para evitar a circulação viral e estas alteraram significativamente o modo de viver dos indivíduos (SILVA HN, et al., 2020; CASAGRANDE M, et al., 2020).

Desde o início da pandemia, foram impostas ações e normas visando reduzir o contato interpessoal nos principais locais de elevado fluxo de pessoas, como nos espaços de convívio comunitário, comércios, empresas, escolas, universidades, transportes públicos, eventos esportivos, culturais e outras situações que possam levar à aglomeração da população. Além disso, o uso de máscaras e os cuidados de higiene foram medidas que mudaram o cotidiano de muitas pessoas e essa nova realidade proporcionou a descontinuação das relações sociais (CASAGRANDE M, et al., 2020).

Nessa situação, sentimentos como o medo, a ansiedade, a tristeza e a preocupação são considerados comuns e compreensíveis que se manifestem nas pessoas. Entretanto, em alguns casos, estas reações podem se prolongar e se agravar, levando a um aumento dos transtornos psíquicos entre homens e mulheres em idade adulta, profissionais de saúde, além de crianças e grupos vulneráveis (OPAS, 2020a; LIMA SO, et al., 2020).

Os transtornos mentais são um grupo de doenças que possuem alto grau de sobrecarga para o indivíduo portador, atingindo também seus familiares e cuidadores. A depressão tem tomado proporções cada vez maiores e pode se tornar, nos próximos 20 anos, a doença mais comum do mundo. Dados da OMS mostram que, em 2020, mais de 350 milhões de pessoas foram afetadas diretamente por essa doença (OPAS, 2020b).

Caracterizada por tristeza persistente, ausência de prazer em atividades normalmente prazerosas, desânimo em realizar atividades cotidianas, baixa autoestima e distúrbios de apetite ou sono, a depressão pode afetar qualquer pessoa em qualquer estágio da vida. Pode, ainda, apresentar sinais e sintomas específicos como irritabilidade, humor deprimido na maior parte do tempo, múltiplas queixas sintomáticas (insônia, anorexia e fadiga), sentimento de culpa excessiva e atraso motor ou agitação (OPAS, 2020b).

A depressão é uma doença multifatorial, que pode ter origem genética, e relaciona-se com a bioquímica cerebral por deficiência de alguns neurotransmissores específicos (noradrenalina, serotonina e dopamina). Esses neurotransmissores associam-se à regulação da atividade motora, sono, apetite e humor e, quando 0 indivíduo já possui uma predisposição genética, atrelado a eventos estressantes, podem ser desencadeados episódios depressivos (BRASIL, 2019). 
Sabe-se ainda que a ansiedade e a depressão estão intimamente relacionadas, porém se diferenciam por sintomatologias diversificadas. Dessa forma, a ansiedade é definida por um sentimento vago e desagradável de medo, que se caracteriza por tensão ou algum desconforto oriundo de antecipação de situações desconhecidas ou estranhas. Ela torna-se patológica a partir do momento que a reação é mais exagerada que o estímulo, podendo ser avaliada de acordo com sua duração, autolimitação e se foi desencadeada pelo estímulo momentâneo ou não (LOPES KP e SANTOS WL, 2018).

Dessa forma, conforme citado anteriormente, todas as medidas restritivas para o controle da disseminação do COVID-19, apesar de absolutamente necessárias e positivas para o controle da doença, acarretaram consequências psicológicas intensas em grande parcela da população (BERARD M, et al., 2021; SILVA HN, et al., 2020). Uma pesquisa feita pela Universidade do Estado do Rio de Janeiro destacou que o número de casos de depressão quase dobraram, enquanto os de ansiedade e estresse aumentaram $80 \%$ no período de quarentena domiciliar. Segundo os resultados do estudo, os problemas de saúde mental estão aumentando em escala preocupante durante a pandemia do COVID-19. O levantamento apontou, ainda, que as mulheres são mais propensas que os homens a sofrer com a ansiedade e estresse durante este o período (FILGEIRAS A e STULTS-KOLEHMAINEN M, 2020; FILGEIRAS A e STULTS-KOLEHMAINEN M, 2021).

Barros MA, et al. (2020), encontraram resultados semelhantes e observaram que cerca de $40,4 \%$ dos brasileiros se sentiram deprimidos ou tristes, e cerca de $52,6 \%$ se sentiram ansiosos ou nervosos durante 0 período da pandemia. Este estudo também constatou que a sensação de tristeza e depressão, bem como a sensação de ansiedade e nervosismo foi, aproximadamente, duas vezes mais prevalente no sexo feminino.

Quando avaliado a vulnerabilidade para sintomas depressivos e sua associação com a idade, um estudo transversal realizado no Rio Grande do Sul demonstrou que adultos jovens, na faixa etária de 18 a 29 anos, apresentaram maior prevalência de sintomas negativos relacionados à saúde mental (DUARTE MQ, et al., 2020). Ademais, as pesquisas têm reforçado que as pessoas com antecedentes ou diagnóstico prévio de depressão são as mais vulneráveis no contexto de pandemia de COVID-19 uma vez que o medo, o estresse e a ansiedade se intensificaram nesses indivíduos durante o período pandêmico (BARROS MA, et al., 2020; BERARD M, et al., 2021; SILVA HN, et al., 2020).

O Ministério da Saúde (MS) também realizou uma pesquisa sobre a saúde mental da população brasileira durante a pandemia da COVID-19. Com relação ao rastreio dos transtornos mentais (ansiedade, depressão e estresse pós-traumático), observou-se $74 \%$ de ansiedade, $26,8 \%$ de depressão na sua forma moderada e $12,3 \%$ na sua forma grave, além de $34,8 \%$ de transtorno de estresse pós-traumático nos brasileiros entrevistados. Foi identificado também que $29,3 \%$ procuraram ajuda profissional por questões relacionadas à saúde mental e 34,2\% informaram que não procuraram ajuda, mas que gostariam de ter apoio psicológico principalmente para lidar com a ansiedade e o estresse (BRASIL, 2021).

Outro importante dado avaliado na pesquisa do MS foi o questionamento sobre consumo de álcool e outras drogas. Os resultados mostraram que $2,2 \%$ dos entrevistados começaram a ingerir álcool durante a pandemia e $16,6 \%$ revelaram aumento no consumo se comparado com o período anterior à pandemia. Além disso, o uso de medicamentos antidepressivos aumentou em $15,8 \%$, sendo que, desses, $7,2 \%$ alegaram que iniciaram a ingestão durante a pandemia. O uso de ansiolíticos foi o que apresentou maior índice (22,6\%) (BRASIL, 2021).

Uma pesquisa realizada pelo Conselho Federal de Farmácia aponta que, no período de janeiro a julho de 2020 , houve um crescimento de quase $14 \%$ nas vendas de antidepressivos e estabilizadores de humor, em comparação com o mesmo período do ano anterior. Foi constatado também que a demanda por fitoterápicos utilizados para o tratamento de ansiedade, como o passiflora, teve um aumento de $59 \%$ no ano de 2020 (CONSELHO FEDERAL DE FARMÁCIA, 2020).

No contexto brasileiro, há ainda uma crise político-institucional com importante impacto econômico gerado pela pandemia, o que propicia o agravamento da ansiedade e insegurança da população no geral. Indivíduos em situações de incerteza sobre emprego e garantia de renda, tendem a apresentar maior risco para o desenvolvimento de transtornos mentais, bem como sentimentos de desamparo, abandono e insegurança 
(NABUCO G, et al., 2020). Um estudo nacional demonstrou que $44,6 \%$ dos trabalhadores entrevistados nas áreas de serviço público, profissionais de saúde, profissionais liberais ou autônomos, bem como profissionais informais tiveram perda econômica nesse período de pandemia, reforçando essa relação entre a insegurança de trabalho e renda com o adoecimento mental da população (DUARTE MQ, 2020).

Outro agravante foi o fato de que pacientes com sintomas de depressão e ansiedade deixaram de procurar ajuda na pandemia e somente uma parte da população recebeu tratamento necessário. Atualmente, a maioria dos diagnósticos de depressão e ansiedade são realizados através de consultas online. Nesses casos, cabe ao profissional coletar dados sobre a história do paciente, levando em conta a história familiar, ambiente em que se vive e experiências anteriores. Em seguida, deve ser realizado um exame de estado mental, observando os comportamentos e sinais do indivíduo. Essas etapas permitem entender o paciente e proceder com o diagnóstico adequado (ZWIELEWSKI G, et al., 2020).

Outro grupo onde é notado um grande impacto na saúde mental são os profissionais da saúde, principalmente aqueles atuantes na linha de frente de enfrentamento ao COVID-19. Com a rotina pesada e o esgotamento mental e físico, os profissionais passam a não ter tempo para cuidar de si próprios e descansar, além de se envolverem diretamente com sentimentos físicos e emoções daqueles para quem prestam serviços, deixando-os ainda mais deprimidos e sobrecarregados. Tem-se observado que muitos destes profissionais manifestam sintomas de Síndrome de Burnout, apresentando maior predomínio de sintomas de depressão e ansiedade (SANTOS KM, et al., 2021).

Outra questão nesse grupo são os baixos salários e baixo reconhecimento profissional de muitos profissionais de saúde, gerando ainda mais inseguranças e fazendo com que, muitos destes, recorram a duplas ou triplas jornadas de trabalho, agravando ainda mais os quadros de desgaste físico e psicológico. Logo, esses profissionais precisam se proteger da fadiga e tensão excessivas, da exaustão resultante da recusa ao descanso e da necessidade de manter a concentração e atenção em alerta para não se contaminarem. Embora estudos apontem resultados menos significativos entre as equipes médicas quando comparados com aqueles obtidos junto à população em geral, é necessário implementar meios para a redução do estresse, da ansiedade, insônia, tristeza, mágoa, desamparo, depressão, frustração e culpa diante das situações vivenciadas diariamente em uma pandemia como a morte de pacientes (SANTOS KM, et al., 2021; ZWIELEWSKI G, et al., 2020).

Em uma pandemia, a prioridade é salvar vidas, bem como fortalecer as medidas de saúde pública e prevenção de agravos. No entanto, as necessidades emocionais das pessoas não podem ser deixadas em segundo plano, caso contrário, podemos estar caminhando para uma outra epidemia, que causará graves danos às pessoas, à sociedade e à economia (OPAS, 2020a). Diante desse cenário, é notório que quaisquer alterações no estado emocional, como o desenvolvimento de ansiedade e depressão, afetam negativamente a vida do indivíduo. Assim, é necessário implementar meios para solucionar este impasse com intuito de que todos os problemas relacionados à perda de saúde mental sejam, no mínimo, minimizados (FARO $\mathrm{A}$, et al., 2020).

Assim, desde o início da pandemia foram necessários reajustes para que os cuidados com a saúde mental não fossem deixados de lado e, com isso, foi autorizado o oferecimento de serviços de forma remota, permitindo a atuação de equipes multiprofissionais da saúde mental, o aumento da comunicação entre os familiares e a criação de uma rede de cuidado para quem já vem apresentando sintomas de transtornos mentais (SCHMIDT B, et al., 2020; SOCCOL KS e SILVEIRA A, 2020).

Dentre as possibilidades de assistência a essa população, tem-se a psicoterapia por meio da rede social com o uso de aplicativos, que estão sendo utilizados de maneira favorável no tratamento do paciente, sendo possível rastrear o histórico clínico e os sintomas dos transtornos mentais atingidos pelos impactos da pandemia. A Terapia Cognitiva Comportamental, também entra como estratégia viável para o auxílio no tratamento de saúde mental podendo ser oferecidas nas Unidades de Saúde da Família, permitindo a identificação de padrões de comportamento e pensamento com o objetivo de criar um plano de tratamento psíquico e a partir disso melhorar o desempenho e humor da população atendida (ZWIELEWSKI G, et al., 2020). 
Ademais, podem ser adotadas medidas como assistência de psicoeducação pelo telefone, planos de valorização do apoio entre os colegas de trabalho e membros da família através de vídeos, bate-papos e ligações, encorajamento à realização de atividades físicas de forma cuidadosa mantendo o distanciamento, bem como incentivo à maiores cuidados com a alimentação (MIRANDA FBG, et al., 2021).

Reforça-se a importância de que, no período de tratamento, seja sempre esclarecido para o paciente sobre a doença e seu quadro clínico, além de incentivá-lo a não desistir do recurso terapêutico. Por fim, é fundamental que os indivíduos com ansiedade e depressão mantenham a conexão com outras pessoas, mesmo dentro da situação atual de confinamento, buscando expressar seus sentimentos e emoções (ZWIELEWSKI G, et al., 2020).

\section{CONSIDERAÇÕES FINAIS}

No decorrer deste estudo foi possível evidenciar não só o impacto direto da pandemia na saúde da população, mas também todos os fatores secundários que têm contribuído para o adoecimento mental dessas pessoas. É notório que as medidas de controle adotadas para conter o avanço da doença contribuíram significativamente para o aumento de casos relacionados à depressão e ansiedade, uma vez que afasta as pessoas de suas rotinas e convívios sociais. É compreensível que nesse momento o objetivo seja enfrentar a pandemia e salvar o maior número de vidas possível, porém existe também a necessidade de se trabalhar de forma paralela para combater essa "pandemia" silenciosa, que tem impactado de forma expressiva a estrutura psicossocial da população, e se não tratada, poderá ocasionar problemas que serão refletidos por décadas em todo mundo, uma vez que essas mudanças psicológicas necessitam de um tempo maior para que se obtenha um resultado de tratamento satisfatório.

\section{REFERÊNCIAS}

1. AFONSO P. O Impacto da Pandemia COVID-19 na Saúde Mental. Revista Científica da Ordem dos Médicos, 2020; 33(5): 356-357.

2. BARROS MA, et. al. Relato de tristeza/depressão, nervosismo/ansiedade e problemas de sono na população adulta brasileira durante a pandemia de COVID-19. Revista Epidemiologia e Serviços de Saúde, 2020; 29(4):e2020427, 2020.

3. BERARD M, et al. Impact of containment and mitigation measures on children and youth with ASD during the COVID19 pandemic: Report from the ELENA cohort. Journal of Psychiatric Research, 2021; 137: 73-80.

4. BRASIL. Ministério da Saúde. Depressão: causas, sintomas, tratamentos, diagnóstico e prevenção. 2019. Disponível em: https://antigo.saude.gov.br/saude-de-a-z/depressao. Acesso em: 10 de maio de 2021.

5. BRASIL. Ministério da Saúde - Coronavirus - COVID-19. 2020. Disponível em: https://coronavirus.saude.gov.br/. Acessado em: 30/04/2021

6. BRASIL. Ministério da Saúde - Segunda etapa da pesquisa inédita realizada pelo Ministério da Saúde abordou procura por atendimento profissional e consumo de álcool e drogas. 2021. Disponível em:https://aps.saude.gov.br/noticia/10658. Acessado em: 20 de maio de 2021.

7. BROOKS SK, et al. The psychological impact of quarantine and how to reduce it: rapid review of the evidence. Lancet, 2020; 395: 912-920.

8. CASAGRANDE M, et al. The enemy who sealed the world: effects quarantine due to the COVID-19 on sleep quality, anxiety, and psychological distress in the Italian population. Sleep Medicine, 2020; 75: 12-20.

9. CONSELHO FEDERAL DE FARMÁCIA. Venda de medicamentos psiquiátricos cresce na pandemia. 2020. Disponível em: http://covid19.cff.org.br/venda-de-medicamentos-psiquiatricos-cresce-na-pandemia/. Acessado em: 20 de maio de 2021.

10. DUARTE MQ, et al. COVID-19 e os impactos na saúde mental: uma amostra do Rio Grande do Sul, Brasil. Ciência e saúde coletiva, 2020; 25(9): 3401-3411.

11. FARO A, et al. COVID-19 e saúde mental: a emergência do cuidado. Estudos de Psicologia (Campinas), 2020; 37: e200074.

12. FILGEIRAS A, STULTS-KOLEHMAINEN M. The Relationship Between Behavioural and Psychosocial Factors Among Brazilians in Quarantine Due to COVID-19. Lancet, 2020; 1-17

13. FILGEIRAS A, STULTS-KOLEHMAINEN M. Risk Factors for Potential Mental Illness Among Brazilians in Quarantine Due to COVID-19. Psychological Reports, 2021; 1-19.

14. FIOCRUZ. Fundação Oswaldo Cruz. Covid-19 Perguntas e respostas. 2020. Disponível em: https://portal.fiocruz.br/pergunta/quais-os-sintomas-do-coronavirus. Acessado em 30/04/2021.

15. LIMA SO, et al. Impactos no comportamento e na saúde mental de grupos vulneráveis em época de enfrentamento da infecção COVID-19: revisão narrativa. Revista Eletrônica Acervo Saúde, 2020; 46: e4006. 
16. LOPES KP, SANTOS WL. Transtorno de ansiedade. Revista de Iniciação Científica, 2018; 1(1): 45-50.

17. MIRANDA FBG, et al. Sofrimento psíquico entre os profissionais de enfermagem durante a pandemia da COVID-19: Scoping Review. Escola Anna Nery, 2021; 25: 1-10

18. NABUCO G, et al. O impacto da pandemia pela COVID-19 na saúde mental: qual é o papel da Atenção Primária à Saúde? Revista Brasileira de Medicina de Família e Comunidade, 2020; 15(42): 2532.

19. ORGANIZAÇÃO MUNDIAL DA SAÚDE (OMS). Mental health and psychosocial considerations during the COVID-19 outbreak. 2020. Disponível em: https://www.who.int/docs/default-source/coronaviruse/mental-healthconsiderations.pdf. Acessado em: 18 de março de 2021.

20. ORGANIZAÇÃO PAN-AMERICANA DE SAÚDE (OPAS). Intervenções recomendadas em saúde mental e apoio psicossocial (Smaps) durante a pandemia. 2020a. Disponível em: https://iris.paho.org/bitstream/handle/10665.2/53017/OPASBRANMHMHCOVID19200026 por.pdf? sequence=1\&isAllowed=y. Acessado em: 19 de maio de 2021.

21. ORGANIZAÇÃO PAN-AMERICANA DE SAÚDE (OPAS). Transtornos Mentais - Principais Fatos, Depressão. $2020 \mathrm{~b}$. Disponível em: https://www.paho.org/pt/topicos/transtornos-mentais. Acessado em: 10 de maio de 2021.

22. SANTOS KR, et al. Depressão e ansiedade em profissionais de enfermagem durante a pandemia da covid-19. Escola Anna Nery, 2021; 25(spe): e20200370.

23. SCHMIDT B, et al. Impacts on Mental Health and Psychological Interventions related to the New Coronavirus Pandemic (COVID-19). Revista Estudos de Psicologia, 2020; 37: 1-13.

24. SILVA HN, et al. Efeitos da pandemia no novo Coronavírus na saúde mental de indivíduos e coletividades. Journal of Nursing and Health, 2020; 10: e20104007.

25. SOCCOL KS, SILVEIRA A. Impactos do distanciamento social na saúde mental: estratégias para a prevenção do suicídio. Journal of Nursing and Health, 2020; 10: e20104033.

26. ZWIELEWSKI G, et al. Protocolos para tratamento psicológico em pandemias: as demandas em saúde mental produzidas pela COVID-19. Revista Debates em Psiquiatria, 2020; 10 (2): 1-8. 